\title{
Flat band in twisted bilayer Bravais lattices
}

\author{
Toshikaze Kariyado $\circledast^{1,2, *}$ and Ashvin Vishwanath ${ }^{2}$ \\ ${ }^{1}$ International Center for Materials Nanoarchitectonics (WPI-MANA), National Institute for Materials Science, Tsukuba 305-0044, Japan \\ ${ }^{2}$ Department of Physics, Harvard University, Cambridge, Massachusetts 02138, USA
}

(Received 23 May 2019; revised manuscript received 22 August 2019; published 5 November 2019)

\begin{abstract}
Band engineering in twisted bilayers of the five generic two-dimensional Bravais networks is demonstrated. We first derive constraints on the interlayer coupling that determines an effective potential for low-energy physics. The constraints are originated from symmetry and fixed without reference to microscopic details. Having the constraints on the potential helps us to predict and understand electron behavior under the influence of the moiré structure, like formation of flat bands. It is also pointed out that the generic constraints become most relevant when the typical length scale of the microscopic interlayer coupling is moderate. The concepts are numerically tested in simple tight-binding models, showing the band flattening compatible with the potential profile fixed by the generic constraints. On the basis of the generic theory, we propose a possibility of anisotropic band flattening, in which quasi-one-dimensional band dispersion is generated from relatively isotropic original band dispersion. In the strongly correlated regime, the anisotropic band flattening leads to a spin-orbital model where intertwined magnetic and orbital ordering can give rise to rich physics. Overall, our theory and demonstration give useful information for screening out interesting materials from the material database.
\end{abstract}

DOI: 10.1103/PhysRevResearch.1.033076

\section{INTRODUCTION}

Having control over electron propagation is a key to derive new functionalities of materials. Under a periodic potential, an energy-momentum dispersion relation of electrons, i.e., a band structure, shows diverse features: one may have a forbidden energy range as a band gap, or the effective mass can differ from the one in vacuum. In crystals, electrons feel the ionic potential, and band engineering is achieved by chemical substitution of ions, for instance. However, the potential profile of ions depends on the properties of the ions themselves given by nature. A possible way to overcome the limitation of the band engineering set by nature is to introduce cleverly designed artificial superstructures into a system [1].

Recently, assembling atomically thin materials [2] has been recognized as an interesting and promising scheme to induce superstructures, especially after the report of correlated electron behaviors and superconductivity in twisted bilayer graphene $[3,4]$. Stacking of two layers can give various superstructures as moiré patterns. When we stack two different layers, a moiré pattern is induced by a lattice constant mismatch. When we stack two identical layers but with a mismatched angle, again we have a corresponding moiré pattern. A moiré pattern induces spatially varying interlayer coupling/interaction, which affects the electron propagation and enables the band engineering [5-9]. Indeed, the correlated

\footnotetext{
*kariyado.toshikaze@nims.go.jp

Published by the American Physical Society under the terms of the Creative Commons Attribution 4.0 International license. Further distribution of this work must maintain attribution to the author(s) and the published article's title, journal citation, and DOI.
}

behaviors in twisted bilayer graphene are associated with effective mass reduction, or band flattening, caused by the moiré pattern $[4,7,8,10,11]$. In general, knowing how interlayer coupling is modulated under a moiré pattern $[12,13]$ is essential to predict electronic structure in the stacked systems.

In this paper, we consider slightly twisted bilayers formed by identical layers of generic two-dimensional Bravais networks: oblique, rectangle, square, diamond (centeredrectangle), and hexagonal networks. We first show that there exist some constraints on the interlayer coupling such that the coupling has to vanish at certain points in the moiré unit cell, depending on which part of the original Brillouin zone the relevant electrons come from. The constraints are of symmetry origin, and derived without reference to the microscopic details of the interlayer coupling. Roughly speaking, vanishing interlayer coupling indicates that the electrons in twisted bilayer feels potential barrier there. Therefore the derived constraints help us to understand and predict how electrons are trapped in the effective potential given by the moiré pattern. Second, by assuming a simple model, we classify the possible situations in terms of the typical length scale of the microscopic interlayer coupling, and identify the regime where the generic constraints become most relevant. The proposed concepts, the constraints and the classification of the regimes, are then numerically demonstrated in simple tightbinding models. We confirm flat band formation ascribed as electron trapping into the effective potential compatible with the constraints. Generally speaking, flat bands are an excellent playground for strong correlation. We show that we can realize nested Hubbard model with a staggered orbital structure on the square lattice, or a spin-valley degenerate Hubbard model in a triangular lattice model. The generic constraints further indicate a notable situation where the band flattening is highly anisotropic, i.e., we can create quasi-one-dimensional bands 
from relatively isotropic bands in each layer. It is shown that anisotropic band flattening leads to an interesting spin-orbital model with intertwined magnetic and orbital coupling in the strongly correlated limit.

Two important ingredients in our theory are the crystalline symmetry and the position of the band top/bottom in the original Brillouin zone. Currently, the majority of the works on twisted bilayers are dealing with hexagonal networks with electrons from $K$ and $K^{\prime}$ points, like graphene, TMDC, and $\mathrm{hBN}$. However, a recent data driven search for exfoliable materials [14] proposes not a few, but hundreds of new candidates. Many of candidates are not hexagonal, which may give a suitable testbed for our theory. Another potential playground is two-dimensional carbon allotropes [15-20]. By changing connections between carbon atoms, various types of networks and a variety of positions of the band top/bottom may be achieved. So, the situation is that we have a large number of candidates from big data or from rich variety of the allotropes. Our theory, the generic constraints and the conditions altogether, gives a toolkit for screening out interesting materials from the candidates.

The paper is organized as follows. Section II is for the basic notions. We explain how the symmetry and the interference of the Bloch phase constrain the interlayer coupling. Also, we derive a condition for the microscopic coupling to make the constraints most powerful in predicting electronic structures. In Sec. III, the basic notions are demonstrated in tight-binding models. As well as the band flattening that follows the symmetry of the original lattice, the anisotropic band flattening is also shown. It is confirmed that the numerical results support the assumptions and the arguments used in building the generic theory. Section IV is devoted to the arguments on possible extensions of the generic theory, the material candidates, and the interesting phenomena associated with the anisotropic band flattening. The paper is summarized in Sec. V.

\section{CONSTRAINTS ON THE INTERLAYER COUPLING}

\section{A. Electrons in moiré pattern}

Let us define the problem: we consider stacking of two identical layers taking the $z$ axis as a normal direction. To have an angle mismatch, the upper layer is rotated by $+\phi / 2$, while the lower layer is rotated by $-\phi / 2$ around the $z$ axis. We assume that each of the layers has the full symmetry of one of the five two-dimensional Bravais lattices. Writing the unit vectors characterizing each layer as $\boldsymbol{a}_{1}$ and $\boldsymbol{a}_{2}$, the moiré pattern induced by twist has a periodicity of $\boldsymbol{L}_{1}$ and $\boldsymbol{L}_{2}$, which are explicitly written as

$$
\boldsymbol{L}_{i}=\hat{z} \times \boldsymbol{a}_{i} /\left(2 \sin \frac{\phi}{2}\right),
$$

i.e., $\boldsymbol{L}_{i}$ is obtained as $90^{\circ}$ rotated $\boldsymbol{a}_{i}$ with the scaling factor $1 /(2 \sin (\phi / 2))$. In a small-angle limit, a system is locally well approximated by an untwisted bilayer, but globally, the moiré pattern is characterized by spatial modulation in the stacking condition. For the later use, we show an explicit formula for the spatial dependence of the stacking condition. In untwisted bilayers, the stacking is described by in-plane relative shift of the two layers, $\boldsymbol{\tau}$. The origin of $\boldsymbol{\tau}$ is defined so that $\boldsymbol{\tau}=0$ corresponds to the right-on-top condition, where the upper layer is derived by the vertical shift of the lower layer. If we set $\boldsymbol{\tau}=0$ on the rotation center, the twist by $\phi$ leads to the spatial dependence of $\boldsymbol{\tau}$ following [12]:

$$
\boldsymbol{\tau}(\boldsymbol{r})=2 \sin \frac{\phi}{2} \hat{z} \times \boldsymbol{r} .
$$

In the following, we use Eq. (2) as it is. Any constant shift of $\boldsymbol{\tau}(\boldsymbol{r}) \rightarrow \boldsymbol{\tau}(\boldsymbol{r})+\boldsymbol{\tau}_{0}$ simply leads to a change in the origin of the moiré pattern, and does not modify the physics in the small-angle limit [8,21]. This global modulation in the stacking condition affects the electronic structure, that is, physical properties can be tuned as a function of the twist angle. The effective model for electrons under the influence of the moiré pattern is generically written as [5,6]

$$
H_{\mathrm{eff}}=\left(\begin{array}{cc}
H_{+}(-i \nabla) & V(\boldsymbol{r}) \\
V^{\dagger}(\boldsymbol{r}) & H_{-}(-i \nabla)
\end{array}\right),
$$

where the diagonal components are for the kinetic energy in each layer with the twist by $\pm \phi / 2$, while the off-diagonal components are for the interlayer coupling. In principle, the diagonal part $\left(H_{ \pm}\right)$can depend on $\boldsymbol{r}$ through the direct static electric potential from the partner layers, but we focus on the case with dominating off-diagonal $V(\boldsymbol{r})$ (as is usually done in graphene). Since the interlayer coupling depends on the local stacking condition, $V(\boldsymbol{r})$ depends on position through the spatial dependence of $\boldsymbol{\tau}(\boldsymbol{r})$. Roughly speaking, in the small-angle or the large moire length scale limit, the kinetic energy containing spatial derivative gets less important. (For instance, when each layer has a quadratic dispersion, the kinetic energy scales as $1 /\left|\boldsymbol{L}_{i}\right|^{2}$ due to $-\nabla^{2}$.) In the regime where the interlayer coupling dominates, it is convenient to go to the basis in which $V(\boldsymbol{r})$ part is diagonalized [12,22]. When each layer is described by a single band model, $V(\boldsymbol{r})$ is a number and we have

$$
\begin{aligned}
\tilde{H}_{\text {eff }}= & \left(\begin{array}{cc}
H_{0}(-i \nabla)+|V(\boldsymbol{r})| & 0 \\
0 & H_{0}(-i \nabla)-|V(\boldsymbol{r})|
\end{array}\right) \\
& + \text { perturbation, }
\end{aligned}
$$

in the basis where $V(\boldsymbol{r})$ becomes diagonal. Here, $H_{0}$ is the average of $H_{+}$and $H_{-}$, and the "perturbation" is from the difference between $H_{+}$and $H_{-}$. This implies that $|V(\boldsymbol{r})|$ plays a role of potential energy. Therefore knowing the properties of $V(\boldsymbol{r})$ is essential to derive the electronic structure in twisted bilayers. The later numerical demonstration supports this interpretation of the interlayer coupling $V(\boldsymbol{r})$ as a potential energy.

As we have noted, a system with small twist is locally well approximated by untwisted bilayers, and therefore, investigating properties of the interlayer coupling in the untwisted cases gives useful information. In the untwisted bilayer, no superstructure is formed and the unit cell is unchanged from the one in each layer. This means that the translation symmetry of the unstacked lattice is kept intact, and the Hamiltonian can be assigned for each momentum $\boldsymbol{k}$ in the original Brillouin zone as [23]

$$
H_{k}=\left(\begin{array}{ll}
H_{0}(\boldsymbol{k}) & V_{k}(\boldsymbol{\tau}) \\
V_{\boldsymbol{k}}^{\dagger}(\boldsymbol{\tau}) & H_{0}(\boldsymbol{k})
\end{array}\right)
$$




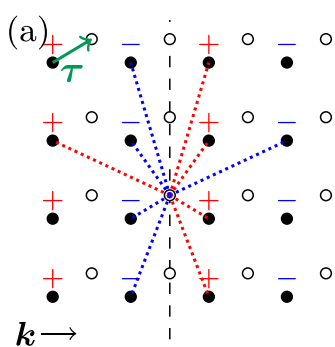

(b)

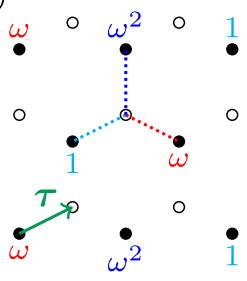

(c)
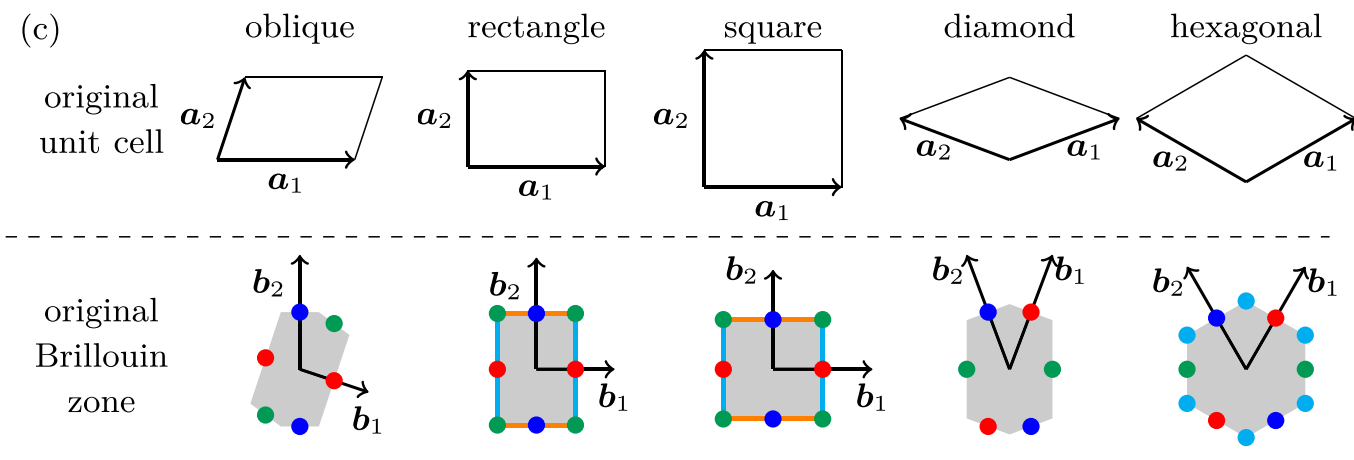

moiré

unit cell
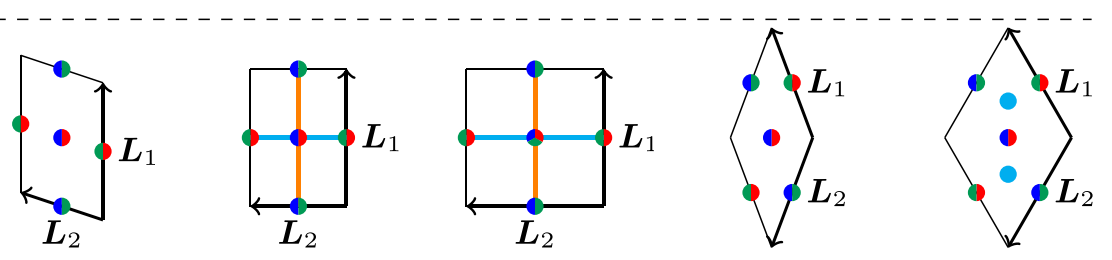

FIG. 1. [(a) and (b)] Examples of the stacking adapted interference caused by intralayer momentum leading to vanishing interlayer coupling, (a) for the square lattice with $\boldsymbol{k}=\boldsymbol{b}_{1} / 2$, and (b) for the triangular lattice with $\boldsymbol{k}= \pm\left(\boldsymbol{b}_{1}+\boldsymbol{b}_{2}\right) / 3$ ( $K$ and $K^{\prime}$ points). (c) Table of the destructive interference manifolds. When the base momentum matches the colored dots or lines in the original Brillouin zones (second row), interlayer coupling vanishes on the dots or lines with the same color in the moiré unit cells (third row). $\boldsymbol{b}_{i}$ in the second line is determined from $\boldsymbol{a}_{i} \cdot \boldsymbol{b}_{j}=2 \pi \delta_{i j}$.

where $H_{0}(\boldsymbol{k})$ is the Hamiltonian for each layer, and the interlayer coupling $V_{\boldsymbol{k}}(\boldsymbol{\tau})$ is now a function of $\boldsymbol{k}$ and $\boldsymbol{\tau}$. To relate $V_{\boldsymbol{k}}(\boldsymbol{\tau})$ with $V(\boldsymbol{r})$, we focus on the situation that the electronic state in each layer is approximated as

$$
H_{\text {single }}=E_{0}+\frac{1}{2} \alpha_{i j} \hat{p}_{i} \hat{p}_{j}, \quad \hat{\boldsymbol{p}}=-i \nabla-\boldsymbol{k}_{0} .
$$

That is, we require each layer to have quadratic band dispersion characterized by an inverse mass tensor $\alpha_{i j}$ around the energy $E_{0}$ and the momentum $\boldsymbol{k}_{0}$. In the following, we call $\boldsymbol{k}_{0}$ the base momentum. In the small-angle limit, the moiré Brillouin zone gets folded and smaller, and usually we look at a few bands in a certain small energy range. If we focus on a small energy window around $E_{0}$, Eq. (6) indicates that the relevant states are mostly contributed from the states with $\boldsymbol{k} \sim \boldsymbol{k}_{0}$ in each layer. In such a case,

$$
V(\boldsymbol{r}) \sim V_{\boldsymbol{k}_{0}}(\boldsymbol{\tau}(\boldsymbol{r}))
$$

should be a good approximation [24]. What is important here is that focusing on a certain energy window fixes the relevant momentum. As far as this correspondence between the energy window and the momentum holds, we can change the explicit form of $H_{\text {single. Since }} V_{\boldsymbol{k}}(\boldsymbol{\tau})$ is periodic in $\boldsymbol{\tau}$ with the period $\boldsymbol{a}_{i}$, while $V(\boldsymbol{r})$ should be periodic in $\boldsymbol{r}$ with the period $\boldsymbol{L}_{i}$, it is convenient to express Eq. (2) using $\boldsymbol{\tau}(\boldsymbol{r})=\tau_{1}(\boldsymbol{r}) \boldsymbol{a}_{1}+\tau_{2}(\boldsymbol{r}) \boldsymbol{a}_{2}$ and $\boldsymbol{r}=r_{1} \boldsymbol{L}_{1}+r_{2} \boldsymbol{L}_{2}$. It reduces to

$$
\tau_{i}(\boldsymbol{r})=-r_{i},
$$

which indicates all possible $\tau$ is contained in a single moiré unit cell spanned by $\left(r_{1}, r_{2}\right) \in[0,1] \otimes[0,1]$.

The expression in Eq. (6) also clarifies the role of $|V(\boldsymbol{r})|$ better. When Eq. (6) leads to a holelike dispersion, or, when we focus on the band top, the highest energy band under the twist will be governed by the component $H_{0}(-i \nabla)+|V(\boldsymbol{r})|$ in Eq. (4). Note that holes are attracted to the region with large $|V(\boldsymbol{r})|$ and avoid the region with small $|V(\boldsymbol{r})|$. On the other hand, when Eq. (6) leads to an electronlike dispersion, or, when we focus on the band bottom, the lowest energy band under the twist will be governed by the component $H_{0}(-i \nabla)-|V(\boldsymbol{r})|$ in Eq. (4). Because of the minus sign in front of $|V(\boldsymbol{r})|$, electrons are attracted to the region with large $|V(\boldsymbol{r})|$ and avoid the region with small $|V(\boldsymbol{r})|$. Importantly, either for a holelike or an electronlike dispersion, manifolds specified by $\{\boldsymbol{r} \mid V(\boldsymbol{r}) \sim 0\}$ will act as potential barriers for holes or electrons.

\section{B. Symmetry-based constraints}

Now, it is worth noting that the symmetry gives constraints on $V_{\boldsymbol{k}}(\boldsymbol{\tau})$. More specifically, for the specific pairs of $(\boldsymbol{k}, \boldsymbol{\tau})$, $V_{k}(\boldsymbol{\tau})$ is guaranteed to vanish. This is originating from an interference of the Bloch phase where symmetry forces exact cancellation of $V_{\boldsymbol{k}}(\tau)$. In the previous work on the untwisted stacking [23], possible sets of $\boldsymbol{k}$ leading to $V_{\boldsymbol{k}}(\boldsymbol{\tau})=0$ are named the stacking $(=\boldsymbol{\tau})$ adapted interference manifold for $\boldsymbol{\tau}$. Let us sketch how the cancellation works in a simple example of a single-orbital tight-binding model on the square lattice. (See Appendix B for the complementary explanation for the cancellation.) In Fig. 1(a), two square networks, depicted by open and closed dots, are stacked with the relative shift $\tau$ [without twist, since we are talking about $V_{k}(\tau)$ ]. Here, we focus on the cancellation given by the reflection line in the vertical direction [like the black dashed line in Fig. 1(a)]. Each square network has two types of vertical reflection lines, one on the line of sites and another in between the line of sites. In Fig. 1(a), $\boldsymbol{\tau}$ is chosen so that the two different types of the reflection lines get on top of each other. Then, a reflection with respect to the dashed line in Fig. 1(a) is a symmetry of the stacked system as well. Now, we consider a case with $\boldsymbol{k}=$ $\boldsymbol{b}_{1} / 2$. [See Fig. 1(c) for the definition of $\boldsymbol{b}_{i}$.] The Bloch phase given by this $\boldsymbol{k}$ is written for the closed dot network (not for the 

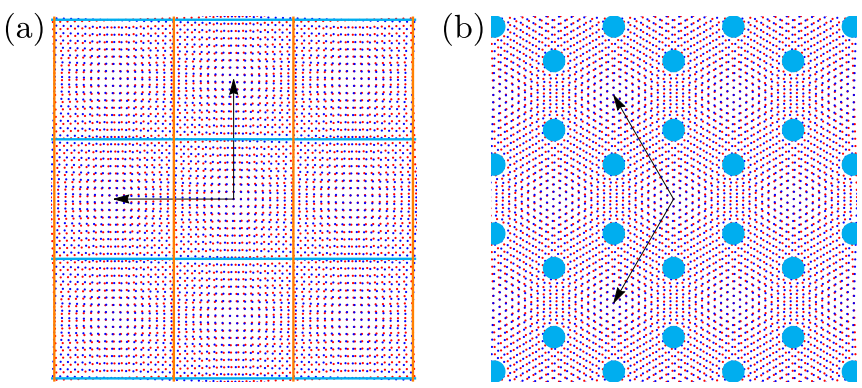

FIG. 2. (a) DIM for the base momentum at the $M$ point in the square lattice. (b) DIM for the base momentum at the $K$ and $K^{\prime}$ points in the triangular lattice.

open dot network to keep the figure simple). We notice that the closed dot network is odd against the reflection with respect to the dashed line, while the open dot network is even against the same operation. This means that the combination of this $\tau$ and $\boldsymbol{k}=\boldsymbol{b}_{1} / 2$ suppresses the interlayer coupling. Alternatively, we may explain this suppression by a cancellation of the microscopic hoppings [dotted lines in Fig. 1(a)] contributing to the interlayer coupling.

In different cases, a rotation axis instead of a reflection plane can also play a pivotal role in canceling the matrix element. Figure 1(b) describes an example in the triangular lattice with $\boldsymbol{k}$ on the $K$ or $K^{\prime}$ point. There, $C_{3}$ rotation makes the contribution by the microscopic hopping [dotted lines in Fig. 1(b)] cancel out $\left(1+\omega+\omega^{2}=0\right.$, where $\omega$ and $\omega^{2}$ are from the Bloch phase for $\boldsymbol{k}$ on the $K$ or $K^{\prime}$ point). Detailed descriptions and a table for the all relevant pairs of $(\boldsymbol{k}, \boldsymbol{\tau})$ in the five two-dimensional Bravais lattices are given in Ref. [23]. Note that $V_{k}(\boldsymbol{\tau})=0$ is derived using generic Bloch states (without degeneracy), and not limited to tight-binding models.

Through Eq. (7), the constraints derived for the untwisted cases impose constraints on the twisted cases as well. If the base momentum $\boldsymbol{k}_{0}$ is on one of the stacking-adapted interference manifold, $V(\boldsymbol{r})$ has to be zero at some point in the moiré unit cell, since a single moiré cell contains all possible $\boldsymbol{\tau}$. We define manifolds (sets of lines or points) specified by $\left\{\boldsymbol{r} \mid V_{\boldsymbol{k}_{0}}(\boldsymbol{\tau}(\boldsymbol{r}))=0\right\}$ as the destructive interference manifold (DIM) for a base momentum $\boldsymbol{k}_{0}$. As we have noted, the lines or points on which $|V(\boldsymbol{r})|=0$ holds behave as potential barriers in the effective model. That is, the shapes of the potential barrier can be fixed by symmetry without reference to microscopic details when the base momentum takes a special value. Figure 1(c) visually summarizes the all DIMs for each of the five Bravais networks. (See Appendix A for the DIMs shown in equations.) The colored manifolds in the moiré unit cell correspond to the DIMs for the base momentum with the same color in the original Brillouin zone. For the selected cases, we also show how the DIM looks like in the extended region with the moiré pattern in Fig. 2.

In Fig. 1(c), the colored dots are associated with the rotation symmetry (mostly $C_{2}$ rotation, except $C_{4}$ as green dots in the square case and $C_{3}$ as cyan dots in the hexagonal case), while the colored lines are associated with the reflection symmetry. In the rectangular and the square lattices, every colored dot is on some colored lines, i.e., the rotation symmetries add nothing than the reflection symmetries give.
However, this information will be useful if we try to extend the arguments here beyond the fully symmetric Bravais networks, or going to the all wallpaper groups where in some cases the reflection planes are removed. The complete discussion on the all wallpaper groups are an interesting future work, but generically, less symmetry will give less restriction, and the fully symmetric cases are most interesting in making meaningful predictions.

\section{Classification of regimes by the typical length scale of the interlayer coupling}

To trap electrons into the effective potential efficiently, having high spatial contrast in $V(\boldsymbol{r})$ is crucial. The constraints by the DIMs are useful in this context since the cancellation of $V(\boldsymbol{r})$ on special points/lines can be a source of high contrast. While the DIMs themselves rely only on symmetry and are generic, having high contrast requires that the spatial range of the microscopic interlayer hopping is moderate compared to the microscopic lattice scale. If the microscopic interlayer coupling is too long-ranged on the scale of the microscopic lattice, an averaging effect suppresses $V(\boldsymbol{r})$ for any $\boldsymbol{r}$ when $\left|\boldsymbol{k}_{0}\right| \neq 0$. On the other hand, if it is too short-ranged, influence of the DIMs originating from the interference of the Bloch phases gets less pronounced since the wave nature is not resolved in very small length scale.

For better understanding, we analyze a simple case that each layer is described by a single-orbital tight-binding model. Here, we write the interlayer hopping as $f(\boldsymbol{r})$ where $\boldsymbol{r}$ is a vector connecting two sites on the different layers projected on the plane of the layer. For a tight-binding model, the wave function is written as a function of the lattice point $\boldsymbol{R}$. Using a gauge where the lower and the upper layer wave functions are written respectively as $\psi_{-}(\boldsymbol{R}) \sim e^{i \boldsymbol{k} \cdot \boldsymbol{R}}$ and $\psi_{+}(\boldsymbol{R}) \sim e^{i \boldsymbol{k} \cdot(\boldsymbol{R}+\boldsymbol{\tau})}, V_{\boldsymbol{k}}(\boldsymbol{r})$ becomes [12,24-28]

$$
V_{\boldsymbol{k}}(\boldsymbol{\tau})=\sum_{\boldsymbol{R}} e^{-i \boldsymbol{k} \cdot(\boldsymbol{R}+\boldsymbol{\tau})} f(\boldsymbol{R}+\boldsymbol{\tau})=\sum_{\boldsymbol{G}} e^{i \boldsymbol{G} \cdot \boldsymbol{\tau}} f_{\boldsymbol{k}+\boldsymbol{G}} .
$$

$f_{\boldsymbol{q}}$ is the Fourier component of $f(\boldsymbol{r})$ and $\boldsymbol{G}$ denotes the reciprocal vectors. When $f(\boldsymbol{r})$ is very long-ranged, $f_{\boldsymbol{q}}$ rapidly decays as a function of $|\boldsymbol{q}|$. But for any $\boldsymbol{k}_{0}$ associated with a DIM, $\left|\boldsymbol{k}_{0}+\boldsymbol{G}\right|$ is always finite and Eq. (9) tell us that $V_{\boldsymbol{k}_{0}}(\boldsymbol{\tau})$ is suppressed as a whole, which means that the associated $V(\boldsymbol{r})$ will be weak anywhere. In addition, if $V_{\boldsymbol{k}_{0}}(\boldsymbol{\tau})$ is suppressed, the spatial dependence of the diagonal part of Eq. (3) may come into play. On the other hand, when $f(\boldsymbol{r})$ is very shortranged, Eq. (9) gives us $\left|V_{k}(\boldsymbol{\tau})\right| \sim|f(\boldsymbol{\tau})|$ with $\boldsymbol{\tau}$ limited in the Wigner-Seitz cell. This does not include any $\boldsymbol{k}$ dependence, i.e., the interference effects are less pronounced. Then, at the intermediate regime where $f(\boldsymbol{r})$ is not too long-ranged nor too short-ranged, the generic constraints derived by symmetry become most relevant. Roughly speaking, in the intermediate regime, the typical length scale should be smaller than the original lattice constant $a$, but still, should be a sizable fraction of $a$. This information gives a useful criterion to pick up interesting materials from the database. Here, we also make a comment on the length scale of $f(\boldsymbol{r})$ in the known examples. Graphene is beyond the assumption in Eq. (6), but when it comes to the range of the interlayer hopping, it is ideal since the estimated $f(\boldsymbol{r})$ is significantly suppressed at 
$|\boldsymbol{r}| \sim\left|\boldsymbol{a}_{i}\right|$, but is sizable, say at $|\boldsymbol{r}|=\left|\boldsymbol{a}_{i}\right| / 2$ [13]. The analysis on $\mathrm{MoS}_{2}$ also indicates that the range of the interlayer hopping is moderate [29].

\section{NUMERICAL DEMONSTRATION}

\section{A. Band flattening}

From now on, the band engineering with the effective potential set by the symmetry-based constraints is demonstrated. Specifically, we show band flattening due to the electron trapping into the effective potential. In the following, band structures for the twisted bilayers are derived using tight-binding models. We work on the twist angles leading to large but commensurate unit cells to have the exact periodicity. For simplicity, the interlayer hopping $f(\boldsymbol{r})$ is assumed to be $[13,30]$

$$
f(\boldsymbol{r})=V_{0} \exp \left(-\frac{r^{2}}{a_{0}^{2}}\right),
$$

where $a_{0}$ controls the effective range of the microscopic interlayer hopping. After adapting this simplification on $f(\boldsymbol{r})$, the tight-binding Hamiltonian is naively solved to obtain the band structure without further approximations. In the previous section for the generic theory, we have assumed that twisted systems are locally well approximated by the untwisted systems. Or, we argued that $V(\boldsymbol{r})$ works as a potential. The following numerical results support these assumptions and arguments. In addition, the argument on the spatial range of $f(\boldsymbol{r})$ is also tested through the $a_{0}$ dependence of the results. In the all examples below, we assume $\left|\boldsymbol{a}_{i}\right|=1$. This means that $a_{0}$ is measured in the unit of $\left|\boldsymbol{a}_{i}\right|$.

The first example is the square lattice model, where the intralayer hoppings are limited to the nearest-neighbor ones of $t=-1$. This leads to the band dispersion

$$
H_{0}(\boldsymbol{k})=2 t\left(\cos k_{1}+\cos k_{2}\right)
$$

in each layer, where $k_{i}=\boldsymbol{k} \cdot \boldsymbol{a}_{i}$. For the interlayer hopping Eq. (10), we set $V_{0}=|t| / 20$. We focus on the state at the band top, which is located at the $M$ point $\left[\boldsymbol{k}=\left(\boldsymbol{b}_{1}+\boldsymbol{b}_{2}\right) / 2\right]$ in the original Brillouin zone. In Fig. 1(c), the $M$ point for the square lattice is on both of the orange and the cyan lines, and correspondingly, the DIM forms square grid lines. [See also Fig. 2(a).] From this, we naively expect that the electrons tend to be trapped in the square lattice avoiding the DIM. In Appendix B, we also analyze $V(\boldsymbol{r})$ for this specific case through Eq. (9).

Figure 3 summarizes the numerical results. Figures 3(b), 3(c), 3(e), and 3(f) are close up views of the energy window near the band top for several combinations of $a_{0}$ and the twist angles. We can clearly see that the top most band pops up and gets flattened as the angle is reduced for $a_{0}=0.6$ in Figs. 3(b) and 3(c). Actually, the top most band is nearly degenerate, but it is difficult to resolve the degeneracy in the shown energy scale. We will return to this degeneracy soon. It is also confirmed that the band flattening gets weaker when $a_{0}$ gets larger in Figs. 3(e) and 3(f). The bandwidth of the top most band is plotted as a function of the moiré length scale $L$ in Fig. 3(d). Note that we originally have quadratic dispersion at the $M$ point, which leads to a trivial contribution to the bandwidth reduction by a factor of $1 / L^{2}$, since the moiré (a) $0^{\circ}, a_{0}=0.8$

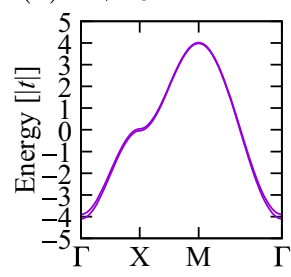

(d)

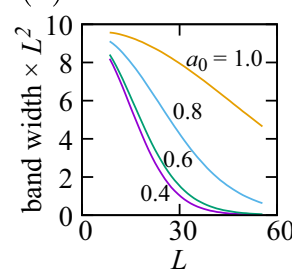

(b) $2.55^{\circ}, a_{0}=0.6$

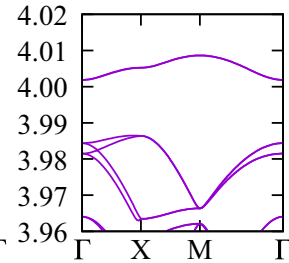

(e) $2.55^{\circ}, a_{0}=1.0$
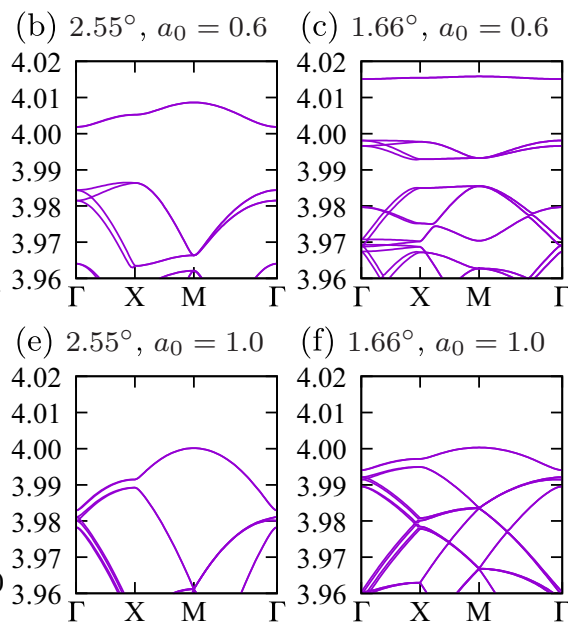

(f) $1.66^{\circ}, a_{0}=1.0$

FIG. 3. (a) Band structure of the untwisted bilayer square lattice model with $a_{0}=0.8$ for $\tau=0$. [(b) and (c)] Band structures of the twisted case with $a_{0}=0.6$ at $\phi=2.55^{\circ}$ and $1.66^{\circ}$, respectively. (d) Band width of the top most band scaled by a factor of $L^{2}$ for several values of $a_{0} . L=30$ and 60 correspond to $\phi=1.91^{\circ}$ and $0.95^{\circ}$, respectively. [(e) and (f)] The same as (b) and (c) but with $a_{0}=1.0$.

Brillouin zone size scales as $1 / L$. Therefore, in Fig. 3(d), the bandwidth is scaled by a factor of $L^{2}$. Still, we observe strong reduction of the scaled bandwidth when $a_{0}$ is small. In this model, the bandwidth times $L^{2}$ roughly corresponds to the effective mass. So, the reduction of the scaled bandwidth is a sign for possible correlated behaviors. In the regime where the band flattening is weak, for instance, when $a_{0}$ is large, it often happens that the bandwidth of the top most band is larger than the energy gap between the top most band and the other bands below as in Figs. 3(e) and 3(f). In such cases, the electron correlation effects will not be pronounced since (i) the bandwidth itself is not small, and (ii) we expect strong screening effects from the nearby bands.

In real space, the flat bands are contributed from one localized orbital per a single moiré unit cell, and the double degeneracy of the top most band comes from the fact that the minimal commensurate supercell for the square moiré pattern contains two moiré unit cells (see Appendix C). The nearest-neighbor pair of moiré cells are microscopically distinguishable, affecting the symmetry of the localized orbitals. Specifically, when we focus on the states from the $M$ point in the original Brillouin zone, the nearest-neighbor pairs are occupied by orbitals with different $C_{4}$ rotation eigenvalues, which we refer to as an $s$-type orbital and a $d_{x y}$-type orbital. [See Fig. 8(c) in Appendix C for the schematic picture.] The different symmetry of the orbitals suppresses the nearestneighbor hopping in moiré cells, and the leading term will be the next nearest-neighbor hopping between the same species of the orbitals, which results in a nested network of the two decoupled square lattices (one from the $s$ orbitals and the other from the $d_{x y}$ orbitals), whose lattice constant is $\sqrt{2}$ of the one of the moiré pattern. Even for incommensurate structures where such a simple $s$ - and $d_{x y}$-orbital picture does not hold, the effect of the nearest-neighbor site decoupling is still apparent in the numerically obtained band structure in the 


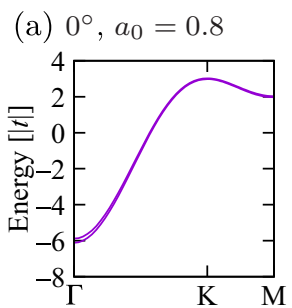

(b) $2.45^{\circ}, a_{0}=0.6$

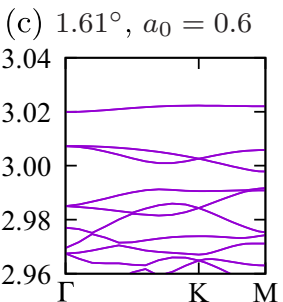

(d)

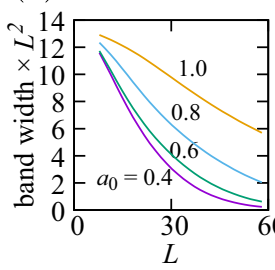

(e) $2.45^{\circ}, a_{0}=1.0$

(f) $1.61^{\circ}, a_{0}=1.0$
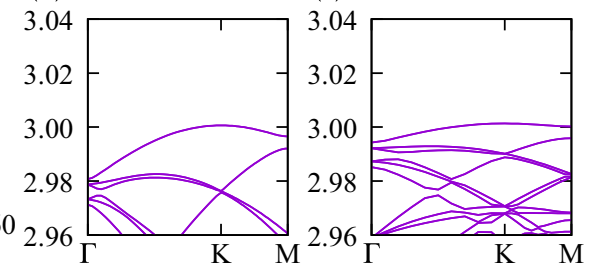

FIG. 4. (a) Band structure of the untwisted bilayer triangular lattice model with $a_{0}=0.8$ for $\tau=0$. We use $V_{0}=|t| / 20$. [(b) and (c)] Band structures of the twisted case with $a_{0}=0.6$ at $\phi=2.45^{\circ}$ and $1.61^{\circ}$, respectively. (d) Band width of the top most band scaled by a factor of $L^{2}$ for several values of $a_{0}$. [(e) and (f)] The same as (b) and (c) but with $a_{0}=1.0$.

small-angle limit (not shown). In the case that the screening from the substrate is strong, electron-electron interaction will be short-ranged in the scale of the moiré pattern, and we expect that the system is modeled as a nested square lattice Hubbard model.

In the square lattice case, the DIM forms grid lines. We can also test the case where the DIM forms arrays of dots, for instance, in the triangular lattice case. If we model each layer by the nearest-neighbor tight-binding model, the band dispersion for the triangular network becomes

$$
H_{0}(\boldsymbol{k})=2 t\left(\cos k_{1}+\cos k_{2}+\cos \left(k_{1}+k_{2}\right)\right)
$$

with $k_{i}=\boldsymbol{k} \cdot \boldsymbol{a}_{i}$. By taking $t=-1$, the band top is at the $K$ and $K^{\prime}$ points $\left[\boldsymbol{k}= \pm\left(\boldsymbol{b}_{1}+\boldsymbol{b}_{2}\right) / 3\right.$ ]. Then, Fig. 1(c) indicates that the DIM forms a honeycomb lattice. [See also Fig. 2(b).] Since the holes avoid these dots, we expect that the holes are trapped in the reciprocal region forming a triangular lattice. Figure 4 summarizes the numerical results for this case, which shows a clear sign of the band flattening for the moderate $a_{0}$. Just as in the case for the square lattice, when $a_{0}$ is small [Figs. 4(b) and 4(c)], the top most bands are well separated from the bands below, while when $a_{0}$ is large, [Figs. 4(e) and 4(f)], it is not the case. Note that the top most bands in Figs. 4(b), 4(c), 4(e), and 4(f) are doubly degenerate, which stems from the degeneracy between the $K$ and $K^{\prime}$ points in the original band structure. For small $a_{0}$, this will give us a Hubbard model on the triangular lattice with extra degrees of freedom from the valley degeneracy in the strongly correlated regime [22,31,32].

\section{B. Anisotropic band flattening}

An interesting application of Fig. 1(c) is that we find situations that the band flattening becomes anisotropic. For
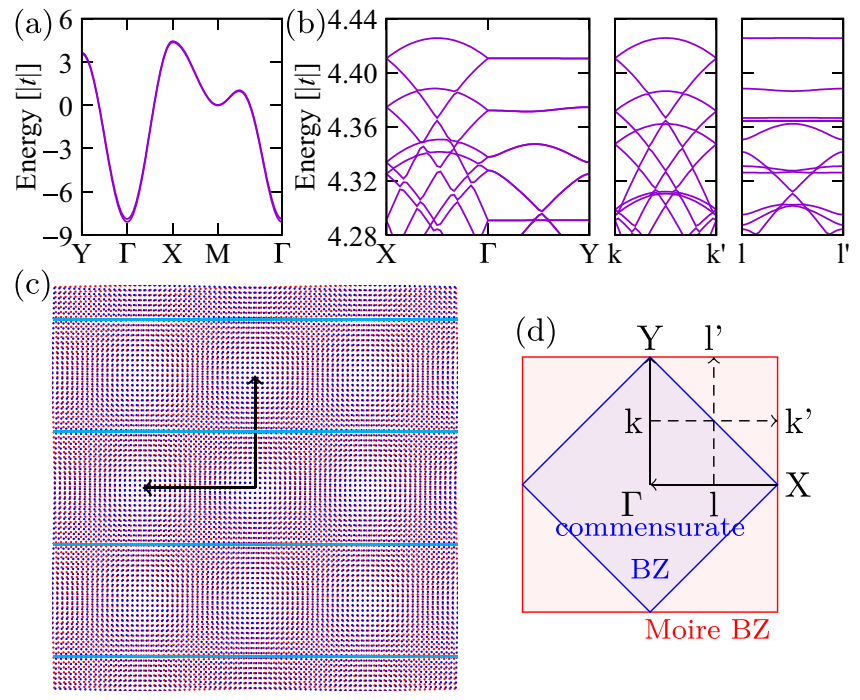

FIG. 5. (a) Band structure of the untwisted bilayer square lattice model with strong n.n.n. hopping. For $\delta<0$, the band top is at the $X$ point $\left(\boldsymbol{k}=\boldsymbol{b}_{1} / 2\right)$. Here, $a_{0}=0.8$ and $\boldsymbol{\tau}=0$ is adapted. (b) Band structure with the twist angle $\phi=2.55^{\circ}, a_{0}=0.6$, and $V_{0}=|t| / 20$ along the path shown in (d). (c) Schematic picture of the destructive interference lines in the moiré structure for the states from the original $X$ point.

the square and the rectangular cases, if the base momentum is on the Brillouin zone edge but not at the zone corner, the DIM runs in one specific direction. This means that the electrons feel quasi-one-dimensional potential. To demonstrate this, we consider the bilayer of a single-orbital tight-binding model on the square lattice with large next nearest-neighbor hoppings. Then, the band dispersion in each layer is modified from Eq. (11) to

$$
\begin{aligned}
H_{0}(\boldsymbol{k})= & 2(t+\delta) \cos k_{1}+2(t-\delta) \cos k_{2} \\
& +2 h\left(\cos \left(k_{x}+k_{y}\right)+\cos \left(k_{x}-k_{y}\right)\right) .
\end{aligned}
$$

By setting $t=-1, h=-1$, and $\delta=-0.1$, the band top locates at the $X$ point $\boldsymbol{k}=\boldsymbol{b}_{1} / 2$ [see Fig. 5(a)]. The finite $\delta$ is introduced simply to make it easier to see the effects by distinguishing the $X$ and $Y$ points. The DIM for this case is schematically written in Fig. 5(c), showing the quasione-dimensional nature. Figure 5(b) shows the obtained band structure under twist along the path in the moiré Brillouin zone specified in Fig. 5(d). We clearly see that the band is flattened in the $y$ direction, while it remains highly dispersive in the $x$ direction, despite the original band structure has only moderate anisotropy around the $X$ point.

A similar anisotropic band flattening is possible also in a triangular lattice model. According to Fig. 1(c), if the base momentum is located on one of the $M$ points, say $\boldsymbol{k}_{0}=$ $\boldsymbol{b}_{2} / 2$, the DIM looks like Fig. 6(c). Since the aspect ratio of the arrays of dots (representing the locations where the interlayer coupling vanishes) is not unity, the anisotropic band flattening is expected. To have the base momentum located on the $M$ point, in each layer we assume hoppings up to the second-nearest-neighbor ones. Then, each of the layers is 


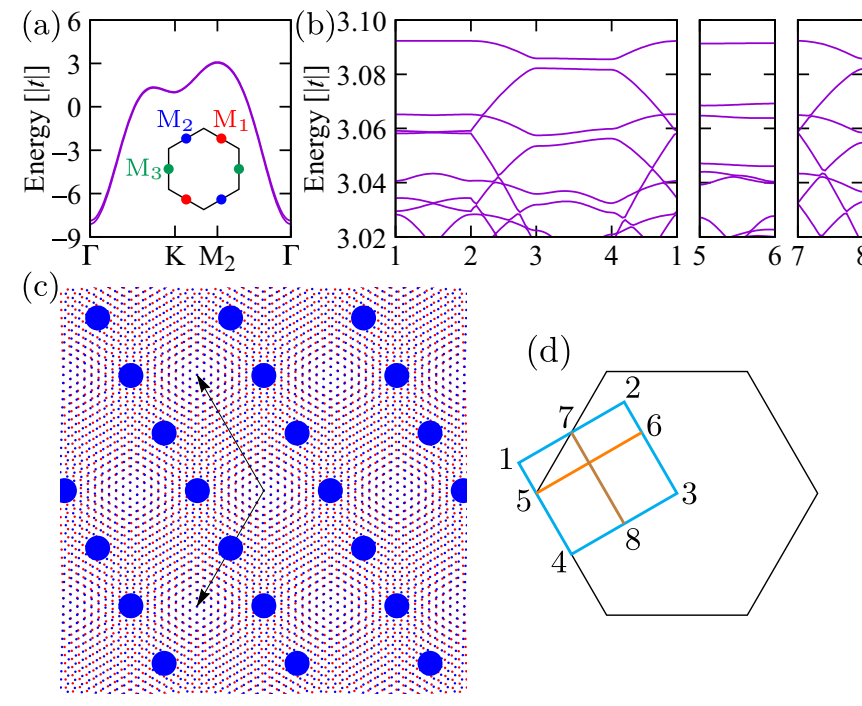

FIG. 6. (a) Band structure of the untwisted bilayer triangular lattice model with n.n. and n.n.n. hopping. For $\delta<0$, the band top is at the $M_{2}$ point $\left(\boldsymbol{k}=\boldsymbol{b}_{2} / 2\right)$. Here, $a_{0}=0.8$ and $\boldsymbol{\tau}=0$ is adapted. (b) Band structure with the twist angle $\phi=2.61^{\circ}, a_{0}=0.6$, and $V_{0}=|t| / 20$. The numbers on the horizontal axis correspond to the points labeled by the same numbers in (d). (c) Schematic picture of the destructive interference lines in the moiré structure for the states from the original $M_{2}$ point.

described by

$$
\begin{aligned}
H_{0}(\boldsymbol{k})= & 2(t+\delta) \cos k_{1}+2(t-\delta) \cos k_{2}+2 t \cos \left(k_{1}+k_{2}\right) \\
& +2 h\left(\cos \left(2 k_{1}+k_{2}\right)+\cos \left(k_{1}+2 k_{2}\right)\right. \\
& \left.+\cos \left(k_{1}-k_{2}\right)\right),
\end{aligned}
$$

instead of Eq. (12). For $t=-1, h=t / 3$, and $\delta=0.1$, the band top is located at the $M_{2}$ point, which can be used as the base momentum [see Fig. 6(a)]. Note that $\delta$ is introduced just for a clear demonstration by isolating one of the three $M$ points. Note also that the band dispersion is isotropic around each of the $M$ point when $\delta=0$. The resultant band structure is shown in Fig. 6(b), where the path depicted in Fig. 6(d) is used. We see that along 1-2, 3-4, and 5-6 directions, the bands are relatively flat, while along $1-4,2-3$, and 7-8 directions, the bands are relatively dispersive. The contrast between the flat and the dispersive parts is most evident in the top most band.

\section{State at the $\Gamma$ point}

We have noted that for very short-ranged $f(\boldsymbol{r})$, the position of the base momentum gets less important and the strong spatial modulation of $V(\boldsymbol{r})$ can be induced without any helps of the DIMs. This statement can be numerically checked by looking at the band bottom at the $\Gamma$ point in the single-orbital tight-binding model of the square and the triangular lattices. It is confirmed that as $a_{0}$ gets reduced, the band flattening gradually becomes prominent (see Appendix D). This means that a material with its base momentum locating on the $\Gamma$ point can be a playground for the correlated physics. However, then, the condition on $a_{0}$ is more stringent than the case utilizing the DIMs.

\section{DISCUSSION}

\section{A. Extension to the multiband cases}

In this paper, we restrict ourselves to the case with a single band in each layer. Practically, this means that the energy gap between the focused band and the others in each layer is larger than the typical energy scale of the interlayer coupling. A possible extension of our theory is to cover the multiband cases. Then, $H_{ \pm}$has multiple components and $V(\boldsymbol{r})$ becomes a matrix as in the well studied case for graphene. We expect that each component of $V(\boldsymbol{r})$ accepts some symmetrybased constraints, but the DIM can be component dependent. This may lead to unique electronic structures. The multiband cases also include an interesting situation where the Berry phase/curvature effects are prominent in each layer. This will be especially important in the case where we have Dirac like linear dispersions in each layer. While we leave generic arguments with linear dispersions (beyond graphene) as a very interesting future problem, we here point out that focusing on the quadratic dispersions has its own merit in persuading correlated physics. In the small-angle limit where the approximation $V(\boldsymbol{r}) \sim V_{\boldsymbol{k}_{0}}(\boldsymbol{\tau}(\boldsymbol{r}))$ is valid, $V(\boldsymbol{r})$ is scale invariant. On the other hand, the kinetic terms scale as $1 / L$ in the linear dispersion case, or as $1 / L^{2}$ in the quadratic dispersion case, where $L$ is the length scale of the moire pattern. Therefore the balance between the kinetic and potential terms in the effective model changes more rapidly with the quadratic dispersions than with the linear dispersions. Then, we expect stronger band flattening at larger twist angles in the quadratic cases comparing with the linear cases.

\section{B. Materials}

The two key ingredients in our demonstration are the crystalline symmetry and the position of the base momentum $\boldsymbol{k}_{0}$. Thus far the most of the studies on the twisted bilayers focused on the hexagonal network with $\boldsymbol{k}_{0}$ at the $K$ and $K^{\prime}$ points, like graphene, TMDC, and hBN. As for the symmetry, among the recently predicted candidates of atomically thin layer materials [14], some of them have square networks, such as square TMD [33] or $\mathrm{Cu}_{2} M X_{4}(M=\mathrm{Mo}, \mathrm{W} ; X=\mathrm{S}, \mathrm{Se})$ [34].

As for the position of $\boldsymbol{k}_{0}, \mathrm{MoS}$ and WS are recently proposed in theory and predicted to have a hexagonal type network with its valence top at the $M$ point [35]. These materials breaks the $C_{2}$-rotation symmetry, which is required for the DIM associated with the $M$ point, but if the symmetry breaking affects the interlayer coupling only perturbatively, the anisotropic band flattening can potentially work. Or, $\gamma$ graphyne, which has a triangular network and has been considered in theory for a long time, is predicted to have the valence top and the conduction bottom at the $M$ point $[15,16]$. This comes with all the required symmetries, but we have to investigate carefully how the internal structure within a single unit cell quantitatively affects the interlayer coupling.

In relation to the anisotropic band flattening, a recent proposal of GeSe is worth to note [36]. There, the anisotropic band flattening is found in the $a b$ initio analysis. Strictly speaking, GeSe is not an ideal material in the DIM-based criterion, but its base momentum is closer to the $X$ point than 


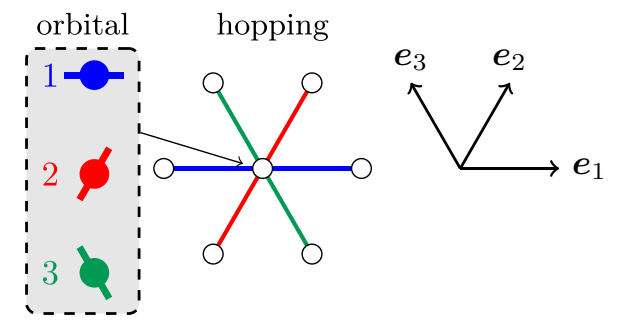

FIG. 7. Schematic picture of the three orbital model.

the $\Gamma$ point, with which we expect the suppression of the interlayer coupling near the DIM to some extent.

\section{Perspectives}

Among the demonstrated results, the anisotropic band flattening is most intriguing and can be of interests in many aspects. In our demonstration of the anisotropic band flattening, the valley degeneracy $(X / Y$ in the square lattice or three $M$ points in the triangular lattice) was deliberately lifted to make the effect easy to be observed. Without that deliberate lifting, we have valley degeneracy, but different valleys result in difference in anisotropy. For convenience, let us define an "easy direction" for each valley as the direction in which the band contributed from the corresponding valley is dispersive. Then, our result is restated as the easy direction depends on the valley. This property might be used to build a valley filter or a valley splitter for valleytronics [37].

On the other hand, with the lifting, or focusing on the rectangular (diamond) lattice instead of the square (triangular) lattice, what we have in the moiré lattice is a set of the quasi-1D channels. In the case that the screening by the substrate is weak, the electron-electron interaction can be long-ranged to couple the neighboring channels. Depending on the parameter, each 1D channel can be nearly free, and then, the entire system can be served as a coupled wire model. Here, the channel distance is tunable by the twist angle. Also, the unit cell is large in the small twist angle cases, which implies that a laboratory accessible magnetic field can amount to a significant fraction of the flux quantum per a unit cell.

Finally, we mention on some novel physics anticipated in the correlated regime with flattened bands. Let us focus on the triangular lattice with base momentum at the $M$ point, where the valley dependent anisotropic band flattening is expected. Under the assumption that the intervalley scattering is weak, which usually applies in the small twist angle limit, three valleys are independent degrees of freedom, and we can build a three orbital model. Because of the anisotropic band flattening, the three orbitals have different easy directions. The simplest model capturing these feature is a three orbital model on the triangular (moiré) lattice, where each orbital only hops between the nearest-neighbor sites in the corresponding easy direction. Figure 7 schematically shows the hopping structure, where the orbital with specific color has only hopping on the bond with the same color. Note that three orbital model in triangular lattice is unique in the sense that there is no three (or higher) dimensional irreducible representation in any $2 \mathrm{D}$ point groups. Neglecting the intervalley scattering is crucial here, since in the symmetry view point, any coupling should lift the three fold degeneracy from the valley degrees of freedom.

As for the correlation, if we assume that the screening by the substrate is strong, the Coulomb interaction is modeled as onsite intra- and interorbital Hubbard repulsion, $U$ and $U^{\prime}$. If we further neglect the Hund coupling and the pair hopping terms, the Hamiltonian becomes,

$$
\begin{aligned}
H= & t \sum_{\boldsymbol{r} \sigma} \sum_{\mu=1}^{3} c_{\boldsymbol{r}+\boldsymbol{e}_{\mu}, \mu \sigma}^{\dagger} c_{\boldsymbol{r}, \mu \sigma}+\text { H.c. } \\
& +U \sum_{\boldsymbol{r}} \sum_{\mu=1}^{3} n_{\boldsymbol{r}, \mu \uparrow} n_{\boldsymbol{r}, \mu \downarrow}+U^{\prime} \sum_{\boldsymbol{r}, \sigma \sigma^{\prime}} \sum_{\mu<\mu^{\prime}} n_{\boldsymbol{r}, \mu \sigma} n_{\boldsymbol{r}, \mu^{\prime} \sigma^{\prime}},
\end{aligned}
$$

where $\boldsymbol{e}_{\mu}$ is defined as in Fig. 7. In the limit of $t \ll U$ and $t \ll U^{\prime}$ with one electron (or hole) per a moiré unit cell, which corresponds to $1 / 6$ filling since we have three orbitals per a site, we can derive a spin-orbital model following the standard $t / U$ expansion. The local basis for the spin-orbital model is $|\sigma\rangle \otimes|\tau\rangle(\sigma=\uparrow, \downarrow, \tau=1,2,3)$. Up to the leading order in $t / U$ and $t / U^{\prime}$, we obtain a variant of the Kugel-Khomskii model [38-40] as

$$
\begin{aligned}
H_{\mathrm{eff}}= & J \sum_{\boldsymbol{r}} \sum_{\mu=1}^{3}\left(\boldsymbol{S}_{\boldsymbol{r}+\boldsymbol{e}_{\mu}} \cdot \boldsymbol{S}_{\boldsymbol{r}}-\frac{1}{4}\right) \tilde{\tau}_{\boldsymbol{r}+\boldsymbol{e}_{\mu}}^{(\mu)} \tilde{\tau}_{\boldsymbol{r}}^{(\mu)} \\
& -V \sum_{\boldsymbol{r}} \sum_{\mu \neq \mu^{\prime}}\left(\tilde{\tau}_{\boldsymbol{r}+\boldsymbol{e}_{\mu}}^{\left(\mu^{\prime}\right)} \tilde{\tau}_{\boldsymbol{r}}^{(\mu)}+\tilde{\tau}_{\boldsymbol{r}-\boldsymbol{e}_{\mu}}^{\left(\mu^{\prime}\right)} \tilde{\tau}_{\boldsymbol{r}}^{(\mu)}\right),
\end{aligned}
$$

with $J=4 t^{2} / U$ and $V=t^{2} / U^{\prime} . \tilde{\tau}^{(\mu)}$ is a $3 \times 3$ matrix acting on the orbital space, whose components are $\left(\tilde{\tau}^{(\mu)}\right)_{i j}=\delta_{i j} \delta_{i \mu}$. Because of the mismatch of the easy direction between the different orbitals, there appears no orbital flip or exchange at this order of the $t / U$-expansion. The orbital dynamics is generated only by higher order terms in the expansion, for instance as a ring exchange term. This means that the orbital degrees of freedom is classical in the Hamiltonian (16), which allows us to derive some eigenstates analytically. For instance, in the case that $V$ dominates, a ground state will be macroscopically degenerate and with maximum short range antiferro-orbital correlation. It is an intriguing future problem to explore the full phase diagram of Eq. (16) and to consider quantum corrections given by the ring exchange terms.

\section{SUMMARY}

To summarize, we derive generic constraints on the interlayer coupling that works as an effective potential for electrons in moiré patterns for bilayers of the all five Bravais networks. The constraints are symmetry-based and fixed without reference to the microscopic details, which helps us to understand and predict how electrons are trapped in an effective potential and form flat bands. Also, it is pointed out that the generic constraints become particularly important when the microscopic interlayer hopping has a moderate length scale compared to the microscopic lattice scale. The power of the constraints is demonstrated in simple tight-binding models by showing band flattening explicitly. From the generic constraints, we find an interesting situation where the band flattening is highly anisotropic. When the interlayer hoppings are 
very short-ranged, the generic constraints are less important, and it is, in principle, possible to have band flattening without constraints. However, having the symmetry-based constraints will boost material searches, and help to build a stable and robust playground for the band flattening. In the presence of interactions, this can provide a promising route to realizing a range of models from quasi-one dimensional coupled wires to square lattice Hubbard models with staggered orbitals, or the Kugel-Khomskii spin orbital models, with a high degree of tunability and control.

\section{ACKNOWLEDGMENTS}

The work was partially supported by JSPS KAKENHI Grants No. JP17K14358 and No. JP18H01162 (T.K.). A.V. was supported by NSF DMR-1411343.

\section{APPENDIX A: DESTRUCTIVE INTERFERENCE MANIFOLD}

In the following, we write the DIM for the base momentum $\boldsymbol{k}_{0}$ in a format $\left\{\boldsymbol{k}_{0}:\right.$ (formula for the DIM) $\}$.

a. Oblique network.

$$
\begin{aligned}
& \left\{\boldsymbol{b}_{1} / 2: \boldsymbol{L}_{1} / 2, \boldsymbol{L}_{1} / 2+\boldsymbol{L}_{2} / 2\right\}, \\
& \left\{\boldsymbol{b}_{2} / 2: \boldsymbol{L}_{2} / 2, \boldsymbol{L}_{1} / 2+\boldsymbol{L}_{2} / 2\right\}, \\
& \left\{\boldsymbol{b}_{1} / 2+\boldsymbol{b}_{2} / 2: \boldsymbol{L}_{1} / 2, \boldsymbol{L}_{2} / 2\right\} .
\end{aligned}
$$

b. Rectangular network.

$$
\begin{aligned}
& \left\{\boldsymbol{b}_{1} / 2: \boldsymbol{L}_{1} / 2, \boldsymbol{L}_{1} / 2+\boldsymbol{L}_{2} / 2\right\}, \\
& \left\{\boldsymbol{b}_{2} / 2: \boldsymbol{L}_{2} / 2, \boldsymbol{L}_{1} / 2+\boldsymbol{L}_{2} / 2\right\}, \\
& \left\{\boldsymbol{b}_{1} / 2+\boldsymbol{b}_{2} / 2: \boldsymbol{L}_{1} / 2, \boldsymbol{L}_{2} / 2\right\}, \\
& \left\{\boldsymbol{b}_{1} / 2+s \boldsymbol{b}_{2}: \boldsymbol{L}_{1} / 2+l \boldsymbol{L}_{2}\right\}(s, l \in[0,1]), \\
& \left\{s \boldsymbol{b}_{1}+\boldsymbol{b}_{2} / 2: l \boldsymbol{L}_{1}+\boldsymbol{L}_{2} / 2\right\}(s, l \in[0,1]) .
\end{aligned}
$$

\section{c. Square network.}

$$
\begin{aligned}
& \left\{\boldsymbol{b}_{1} / 2: \boldsymbol{L}_{1} / 2, \boldsymbol{L}_{1} / 2+\boldsymbol{L}_{2} / 2\right\}, \\
& \left\{\boldsymbol{b}_{2} / 2: \boldsymbol{L}_{2} / 2, \boldsymbol{L}_{1} / 2+\boldsymbol{L}_{2} / 2\right\}, \\
& \left\{\boldsymbol{b}_{1} / 2+\boldsymbol{b}_{2} / 2: \boldsymbol{L}_{1} / 2, \boldsymbol{L}_{2} / 2, \boldsymbol{L}_{1} / 2+\boldsymbol{L}_{2} / 2\right\}, \\
& \left\{\boldsymbol{b}_{1} / 2+s \boldsymbol{b}_{2}: \boldsymbol{L}_{1} / 2+l \boldsymbol{L}_{2}\right\}(s, l \in[0,1]), \\
& \left\{s \boldsymbol{b}_{1}+\boldsymbol{b}_{2} / 2: l \boldsymbol{L}_{1}+\boldsymbol{L}_{2} / 2\right\}(s, l \in[0,1]) .
\end{aligned}
$$

\section{d. Diamond network.}

$$
\begin{aligned}
& \left\{\boldsymbol{b}_{1} / 2: \boldsymbol{L}_{1} / 2, \boldsymbol{L}_{1} / 2+\boldsymbol{L}_{2} / 2\right\}, \\
& \left\{\boldsymbol{b}_{2} / 2: \boldsymbol{L}_{2} / 2, \boldsymbol{L}_{1} / 2+\boldsymbol{L}_{2} / 2\right\}, \\
& \left\{\boldsymbol{b}_{1} / 2+\boldsymbol{b}_{2} / 2: \boldsymbol{L}_{1} / 2, \boldsymbol{L}_{2} / 2\right\} .
\end{aligned}
$$

e. Hexagonal network.

$$
\begin{aligned}
& \left\{\boldsymbol{b}_{1} / 2: \boldsymbol{L}_{1} / 2, \boldsymbol{L}_{1} / 2+\boldsymbol{L}_{2} / 2\right\}, \\
& \left\{\boldsymbol{b}_{2} / 2: \boldsymbol{L}_{2} / 2, \boldsymbol{L}_{1} / 2+\boldsymbol{L}_{2} / 2\right\}, \\
& \left\{\boldsymbol{b}_{1} / 2+\boldsymbol{b}_{2} / 2: \boldsymbol{L}_{1} / 2, \boldsymbol{L}_{2} / 2\right\}, \\
& \left\{\boldsymbol{b}_{1} / 3+\boldsymbol{b}_{2} / 3: 2 \boldsymbol{L}_{1} / 3+\boldsymbol{L}_{2} / 3, \boldsymbol{L}_{1} / 3+2 \boldsymbol{L}_{2} / 3\right\}, \\
& \left\{-\boldsymbol{b}_{1} / 3-\boldsymbol{b}_{2} / 3: 2 \boldsymbol{L}_{1} / 3+\boldsymbol{L}_{2} / 3, \boldsymbol{L}_{1} / 3+2 \boldsymbol{L}_{2} / 3\right\} .
\end{aligned}
$$

\section{APPENDIX B: ANALYSIS OF EQ. (9)}

Let us check the behavior of $V_{k}(\boldsymbol{\tau})$ through Eq. (9). Just for a demonstration purpose, we further assume Eq. (10) for $f(\boldsymbol{r})$, which leads to

$$
f_{\boldsymbol{k}+\boldsymbol{G}} \propto \exp \left(-\frac{a_{0}^{2}|\boldsymbol{k}+\boldsymbol{G}|^{2}}{4}\right) .
$$

Now, we focus on the square lattice case with $\boldsymbol{k}$ at the $M$ point $\left(\boldsymbol{k}=\left(\boldsymbol{b}_{1}+\boldsymbol{b}_{2}\right) / 2\right)$. In this case, the minimum $|\boldsymbol{k}+\boldsymbol{G}|$ is $\left|\boldsymbol{b}_{1}\right| / \sqrt{2}=\left|\boldsymbol{b}_{2}\right| / \sqrt{2}$. (Note that $\boldsymbol{G}$ is generically written as $\boldsymbol{G}=n_{1} \boldsymbol{b}_{1}+n_{2} \boldsymbol{b}_{2}$ with integers $n_{1}$ and $n_{2}$.) As a rough approximation to capture the physics behind the cancellation of $V_{\boldsymbol{k}}(\boldsymbol{\tau})$, we leave only the contributions from $\boldsymbol{G}$ leading to $|\boldsymbol{k}+\boldsymbol{G}|=\left|\boldsymbol{b}_{1}\right| / \sqrt{2}$. Then, Eq. (9) gives

$$
V_{\boldsymbol{k}}(\boldsymbol{\tau})=f_{\left|\boldsymbol{b}_{1}\right| / \sqrt{2}}\left(1+e^{-i \boldsymbol{b}_{1} \cdot \boldsymbol{\tau}}\right)\left(1+e^{-i \boldsymbol{b}_{2} \cdot \boldsymbol{\tau}}\right) .
$$

From this and Eq. (8), the DIM can be derived by the condition $e^{i \boldsymbol{b}_{1,2} \cdot \boldsymbol{\tau}(\boldsymbol{r})}=-1$, which ends up with Fig. 2(a). As far as we adapt the simple form of Eq. (10), it is easy to derive the DIMs for the other cases as well.

\section{APPENDIX C: COMMENSURATE UNIT CELL FOR THE SQUARE LATTICE}

An easy and straightforward way to derive band structures with moiré patterns is to use a commensurate unit cell, which is large and strictly periodic. For the square lattice case, the unit vectors for the minimal commensurate cell with twist $\tilde{\boldsymbol{L}}_{i}$ are derived using the original unit vectors $\boldsymbol{a}_{i}$ as

$$
\begin{gathered}
\tilde{\boldsymbol{L}}_{1}=(s+1) \boldsymbol{a}_{1}+s \boldsymbol{a}_{2}, \\
\tilde{\boldsymbol{L}}_{2}=-s \boldsymbol{a}_{1}+(s+1) \boldsymbol{a}_{2}
\end{gathered}
$$

with $s$ an integer and $2 \sin (\phi / 2)=1 / \sqrt{s^{2}+s+1 / 2}$. However, $\left|\tilde{\boldsymbol{L}}_{i}\right|=\sqrt{2}\left|\boldsymbol{L}_{i}\right|$ and the area spanned by $\tilde{\boldsymbol{L}}_{1}$ and $\tilde{\boldsymbol{L}}_{2}$ is twice as large as the one spanned by $\boldsymbol{L}_{1}$ and $\boldsymbol{L}_{2}$. Typical commensurate and incommensurate moiré patterns are shown in Fig. 8, where the moiré period is indicated by grid lines. For the commensurate case, in the moiré cell at the center, we have dots on the crossing of the grid lines. On the other hand, in the neighboring moiré cells, there are no dots on the crossing of the grid lines. That is, there are two types of microscopically distinguishable moiré cells. 

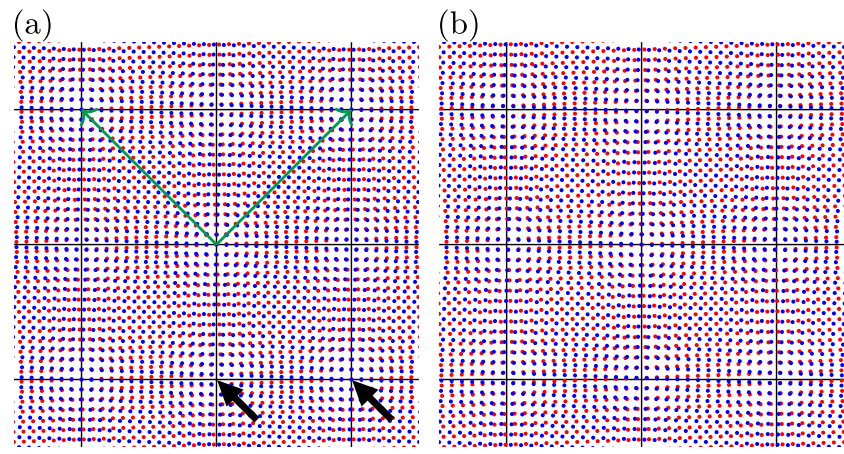

(c)
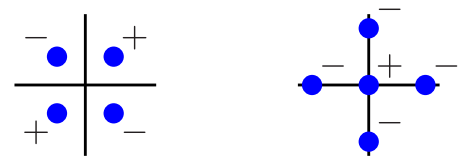

FIG. 8. (a) Moiré pattern at the angle satisfying the minimal commensurate condition. The moiré period is indicated by grid lines, while the commensurate unit vectors are written as arrows. At the crossing of the grid lines, the stacking condition is $\tau=0$, but some crossings have sites right on top, while the others do not, i.e., two regions with the same $\boldsymbol{\tau}=0$ are microscopically distinguishable. (b) Moiré pattern at the incommensurate angle to compare with (a). (c) Schematic description for the phase structure of the wave function near the points marked by black arrows in (a). The momentum at the $M$ point $\left[\boldsymbol{k}=\left(\boldsymbol{b}_{1}+\boldsymbol{b}_{2}\right) / 2\right]$ gives this sign structure.

\section{APPENDIX D: STATE AT THE $\Gamma$ POINT}

It often happens that the base momentum is located at the $\Gamma$ point. However, the $\Gamma$ point, which is the center of the
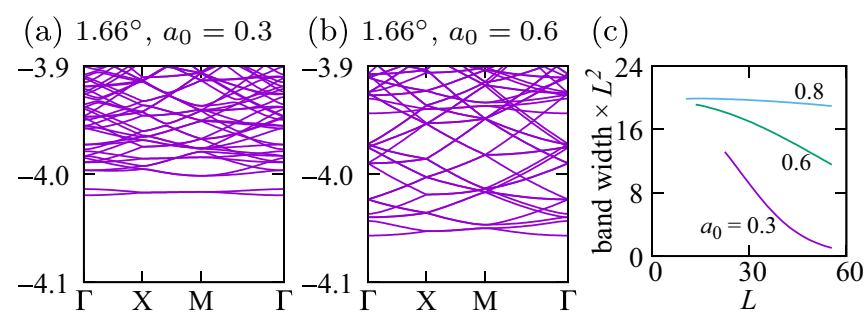

(d) $1.61^{\circ}, a_{0}=0.3$

(e) $1.61^{\circ}, a_{0}=0.6$
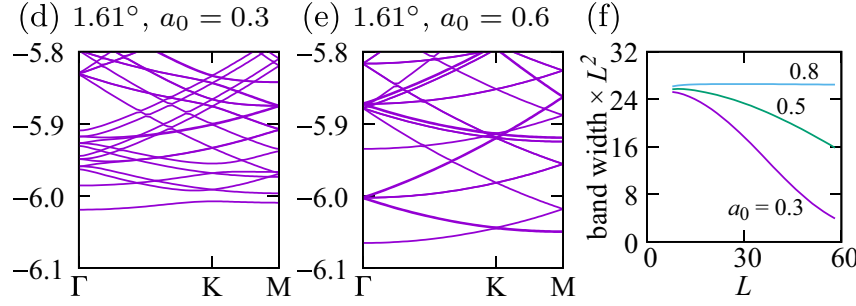

FIG. 9. Band structures of the twisted bilayer square lattice model with (a) $a_{0}=0.3$ and 0.6. The lower most bands are featured to see the case that the base momentum is located at the $\Gamma$ point. (c) Scaled bandwidth of the lower most band. [(d)-(f)] The same as (a)-(c) but for the triangular lattice model.

Brillouin zone, is not related to any of the DIMs as Fig. 1(c) shows. This means that the generic constraints cannot fix the shape of the effective potential barriers, and we have to have very short-ranged interlayer hopping. To demonstrate the band flattening at the $\Gamma$ point, we focus on the band bottom of the nearest-neighbor tight-binding models on the square and the triangular lattices. Figure 9 summarizes the numerical results, for which the set up is the same as the one for Figs. 3 and 4. We find that the band flattening is prominent only for small $a_{0}$.
[1] L. Esaki and R. Tsu, Superlattice and negative differential conductivity in semiconductors, IBM J. Res. Dev. 14, 61 (1970).

[2] A. K. Geim and I. V. Grigorieva, Van der Waals heterostructures, Nature (London) 499, 419 (2013).

[3] Y. Cao, V. Fatemi, A. Demir, S. Fang, S. L. Tomarken, J. Y. Luo, J. D. Sanchez-Yamagishi, K. Watanabe, T. Taniguchi, E. Kaxiras, R. C. Ashoori, and P. Jarillo-Herrero, Correlated insulator behaviour at half-filling in magic-angle graphene superlattices, Nature (London) 556, 80 (2018).

[4] Y. Cao, V. Fatemi, S. Fang, K. Watanabe, T. Taniguchi, E. Kaxiras, and P. Jarillo-Herrero, Unconventional superconductivity in magic-angle graphene superlattices, Nature (London) 556, 43 (2018).

[5] J. M. B. Lopes dos Santos, N. M. R. Peres, and A. H. Castro Neto, Graphene Bilayer with a Twist: Electronic Structure, Phys. Rev. Lett. 99, 256802 (2007).

[6] E. J. Mele, Commensuration and interlayer coherence in twisted bilayer graphene, Phys. Rev. B 81, 161405(R) (2010).

[7] E. Suárez Morell, J. D. Correa, P. Vargas, M. Pacheco, and Z. Barticevic, Flat bands in slightly twisted bilayer graphene: Tight-binding calculations, Phys. Rev. B 82, 121407(R) (2010).

[8] R. Bistritzer and A. H. MacDonald, Moiré bands in twisted double-layer graphene, Proc. Natl. Acad. Sci. USA 108, 12233 (2011).
[9] M. Koshino, Interlayer interaction in general incommensurate atomic layers, New J. Phys. 17, 015014 (2015).

[10] N. N. T. Nam and M. Koshino, Lattice relaxation and energy band modulation in twisted bilayer graphene, Phys. Rev. B 96, 075311 (2017).

[11] G. Tarnopolsky, A. J. Kruchkov, and A. Vishwanath, Origin of Magic Angles in Twisted Bilayer Graphene, Phys. Rev. Lett. 122, 106405 (2019).

[12] J. Jung, A. Raoux, Z. Qiao, and A. H. MacDonald, Ab initio theory of moiré superlattice bands in layered two-dimensional materials, Phys. Rev. B 89, 205414 (2014).

[13] S. Fang and E. Kaxiras, Electronic structure theory of weakly interacting bilayers, Phys. Rev. B 93, 235153 (2016).

[14] N. Mounet, M. Gibertini, P. Schwaller, D. Campi, A. Merkys, A. Marrazzo, T. Sohier, I. E. Castelli, A. Cepellotti, G. Pizzi, and N. Marzari, Two-dimensional materials from high-throughput computational exfoliation of experimentally known compounds, Nat. Nanotechnol. 13, 246 (2018).

[15] R. H. Baughman, H. Eckhardt, and M. Kertesz, Structureproperty predictions for new planar forms of carbon: Layered phases containing $s p^{2}$ and $s p$ atoms, J. Chem. Phys. 87, 6687 (1987). 
[16] N. Narita, S. Nagai, S. Suzuki, and K. Nakao, Optimized geometries and electronic structures of graphyne and its family, Phys. Rev. B 58, 11009 (1998).

[17] H. Lu and S.-D. Li, Two-dimensional carbon allotropes from graphene to graphyne, J. Mater. Chem. C 1, 3677 (2013).

[18] Y. Li, L. Xu, H. Liu, and Y. Li, Graphdiyne and graphyne: From theoretical predictions to practical construction, Chem. Soc. Rev. 43, 2572 (2014).

[19] L.-C. Xu, R.-Z. Wang, M.-S. Miao, X.-L. Wei, Y.-P. Chen, H. Yan, W.-M. Lau, L.-M. Liu, and Y.-M. Ma, Two dimensional Dirac carbon allotropes from graphene, Nanoscale 6, 1113 (2014).

[20] Z. Jia, Y. Li, Z. Zuo, H. Liu, C. Huang, and Y. Li, Synthesis and properties of 2D carbon-graphdiyne, Acc. Chem. Res. 50, 2470 (2017).

[21] L. Zou, H. C. Po, A. Vishwanath, and T. Senthil, Band structure of twisted bilayer graphene: Emergent symmetries, commensurate approximants, and Wannier obstructions, Phys. Rev. B 98, 085435 (2018).

[22] F. Wu, T. Lovorn, E. Tutuc, and A. H. MacDonald, Hubbard Model Physics in Transition Metal Dichalcogenide Moiré Bands, Phys. Rev. Lett. 121, 026402 (2018).

[23] R. Akashi, Y. Iida, K. Yamamoto, and K. Yoshizawa, Interference of the Bloch phase in layered materials with stacking shifts, Phys. Rev. B 95, 245401 (2017).

[24] M. Koshino, N. F. Q. Yuan, T. Koretsune, M. Ochi, K. Kuroki, and L. Fu, Maximally Localized Wannier Orbitals and the Extended Hubbard Model for Twisted Bilayer Graphene, Phys. Rev. X 8, 031087 (2018).

[25] J. M. B. Lopes dos Santos, N. M. R. Peres, and A. H. Castro Neto, Continuum model of the twisted graphene bilayer, Phys. Rev. B 86, 155449 (2012).

[26] P. Moon and M. Koshino, Optical absorption in twisted bilayer graphene, Phys. Rev. B 87, 205404 (2013).

[27] Y. Wang, Z. Wang, W. Yao, G.-B. Liu, and H. Yu, Interlayer coupling in commensurate and incommensurate bilayer structures of transition-metal dichalcogenides, Phys. Rev. B 95, 115429 (2017).

[28] F. Rost, R. Gupta, M. Fleischmann, D. Weckbecker, N. Ray, J. Olivares, M. Vogl, S. Sharma, O. Pankratov, and S. Shallcross,
A non-perturbative theory of effective Hamiltonians: example of moiré materials, arXiv:1901.04535.

[29] S. Fang, R. Kuate Defo, S. N. Shirodkar, S. Lieu, G. A. Tritsaris, and E. Kaxiras, Ab initio tight-binding Hamiltonian for transition metal dichalcogenides, Phys. Rev. B 92, 205108 (2015).

[30] B. Padhi and P. W. Phillips, Pressure-induced metal-insulator transition in twisted bilayer graphene, Phys. Rev. B 99, 205141 (2019).

[31] F. Wu, T. Lovorn, E. Tutuc, I. Martin, and A. H. MacDonald, Topological Insulators in Twisted Transition Metal Dichalcogenide Homobilayers, Phys. Rev. Lett. 122, 086402 (2019).

[32] L. Xian, D. M. Kennes, N. Tancogne-Dejean, M. Altarelli, and A. Rubio, Multi-flat bands and strong correlations in twisted bilayer boron nitride, Nano Lett. 19, 4934 (2019).

[33] L.-Y. Gan and U. Schwingenschlögl, Two-dimensional square ternary $\mathrm{Cu}_{2} M X_{4}(M=\mathrm{Mo}, \mathrm{W} ; X=\mathrm{S}, \mathrm{Se})$ monolayers and nanoribbons predicted from density functional theory, Phys. Rev. B 89, 125423 (2014).

[34] Y. Ma, L. Kou, X. Li, Y. Dai, S. C. Smith, and T. Heine, Quantum spin Hall effect and topological phase transition in two-dimensional square transition-metal dichalcogenides, Phys. Rev. B 92, 085427 (2015).

[35] D. Pandey and A. Chakrabarti, Prediction of two-dimensional monochalcogenides: MoS and WS, Phys. Lett. A 383, 2914 (2019).

[36] D. M. Kennes, L. Xian, M. Claassen, and A. Rubio, A new twist in the realization of one-dimensional physics, arXiv: 1905.04025 .

[37] A. Rycerz, J. Tworzydlo, and C. W. J. Beenakker, Valley filter and valley valve in graphene, Nat. Phys. 3, 172 (2007).

[38] K. I. Kugel and D. I. Khomskiŭ, The Jahn-Teller effect and magnetism: transition metal compounds, Sov. Phys. Usp. 25, 231 (1982).

[39] G. Khaliullin and V. Oudovenko, Spin and orbital excitation spectrum in the Kugel-Khomskii model, Phys. Rev. B 56, R14243 (1997).

[40] F. Wang and A. Vishwanath, $Z_{2}$ spin-orbital liquid state in the square lattice Kugel-Khomskii model, Phys. Rev. B 80, 064413 (2009). 